\title{
A custom microarray platform for analysis of microRNA gene expression
}

\author{
J Michael Thomson $^{1}$, Joel Parker ${ }^{2,5}$, Charles M Perou ${ }^{2-4}$ \& Scott M Hammond ${ }^{1,2}$
}

\begin{abstract}
MicroRNAs are short, noncoding RNA transcripts that posttranscriptionally regulate gene expression. Several hundred microRNA genes have been identified in Caenorhabditis elegans, Drosophila, plants and mammals. MicroRNAs have been linked to developmental processes in $C$. elegans, plants and humans and to cell growth and apoptosis in Drosophila. A major impediment in the study of microRNA function is the lack of quantitative expression profiling methods. To close this technological gap, we have designed dual-channel microarrays that monitor expression levels of 124 mammalian microRNAs. Using these tools, we observed distinct patterns of expression among adult mouse tissues and embryonic stem cells. Expression profiles of staged embryos demonstrate temporal regulation of a large class of microRNAs, including members of the let-7 family. This microarray technology enables comprehensive investigation of microRNA expression, and furthers our understanding of this class of recently discovered noncoding RNAs.
\end{abstract}

MicroRNAs comprise a large family of noncoding RNAs found in organisms ranging from nematodes to plants to humans (see ref. 1 for a review). Over 200 microRNAs have been identified in mammals, either through computational searches or by RT-PCRmediated cloning. These RNAs function as natural triggers of the RNAi pathway, regulating gene expression at a post-transcriptional step.

MicroRNA biogenesis begins with a primary transcript that contains a stem-loop structure ${ }^{1}$. This transcript is processed by the ribonuclease III enzyme Drosha, liberating the stem-loop, which is termed the precursor. This precursor is transported out of the nucleus in a process dependent on the Ran GTPase and the export receptor exportin-5. Further processing in the cytoplasm by the ribonuclease III enzyme Dicer leads to the production of mature RNAs of $\sim 22$ nucleotides (nt) that are incorporated into the RNAi effector complex RISC (RNA-induced silencing complex). Complementarity with elements in mRNAs leads to suppression of gene expression. In cases where the microRNA is an imperfect match to the mRNA, as with C. elegans lin-4, recognition leads to reduction in protein levels without affecting mRNA levels. In plants, mRNA targets in the scarecrow-like family of transcription factors contain sequences perfectly complementary to the microRNA miR-39. Similarly, in mammals, miR-196 has near-perfect identity with elements in the mRNA of the homeobox transcription factor gene HoxB8 (ref. 2). In this case recognition of the mRNA by microRNAs leads to cleavage, rather than translational repression, analogous to siRNA-mediated gene silencing ${ }^{3,4}$.

Despite the large number of identified microRNAs, the scope of their roles in regulating cellular gene expression is not known. The founding members of this family of noncoding RNAs are the C. elegans lin-4 and let-7 (refs. 5,6). Expression of these microRNAs, originally termed short-temporal RNAs, is essential for proper timing of events during larval development. For example, levels of the let-7 RNA increase during the fourth larval stage and the adult stage, resulting in suppression of larval-specific genes, including lin-41 (ref. 6). Partially complementary elements in the lin-41 mRNA are binding sites for let-7 (ref. 7). The role of microRNAs in cell lineage and development has recently been found to extend to mammalian systems. miR-181 is highly expressed in hematopoietic progenitors, and its overexpression promotes differentiation into B-lineage cells ${ }^{8}$. The regulation of homeobox genes by microRNAs further links this gene family to mammalian developmental processes ${ }^{2}$.

One approach to identifying the cellular roles of microRNAs is the identification of mRNA targets. Several groups have developed computational methods to search for target sequences of microRNAs (see ref. 1 for a discussion). These methods have yielded hundreds of candidate targets in plants, Drosophila and mammals that implicate microRNAs in a diverse range of cellular pathways. Essential for the interpretation of these data, however, is an understanding of microRNA expression patterns vis-à-vis expression patterns of predicted targets. The temporally restricted expression of large sets of microRNAs in C. elegans and Drosophila has been reported ${ }^{9-11}$. More recently, tissue-specific expression patterns of mammalian microRNAs have been described ${ }^{12}$. All data were obtained by northern blot analysis of microRNA levels. As a refinement to this approach, the use of nylon macroarrays for analysis of 44 microRNAs during brain development has been reported ${ }^{13}$. All the aforementioned approaches, however, 

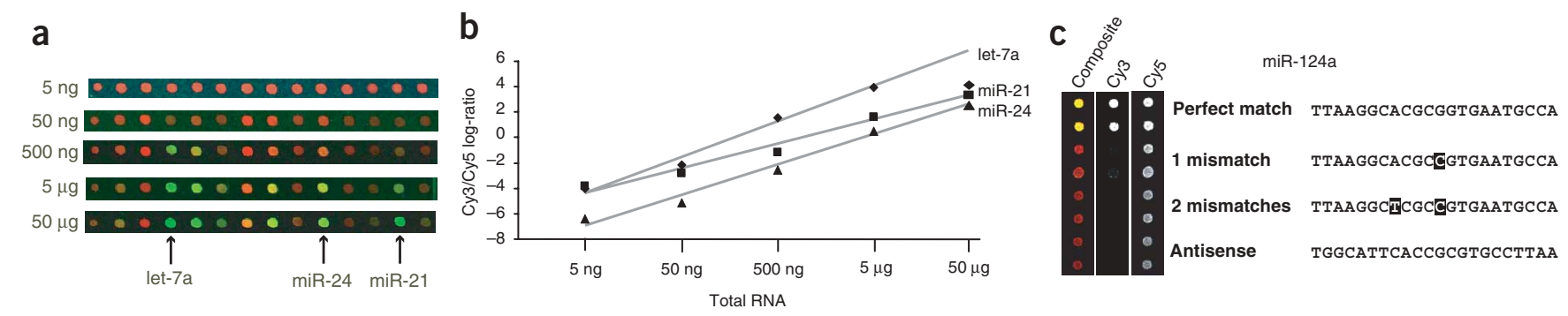

Figure 2 | Sensitivity and specificity of microRNA microarrays. (a) Total RNA from mouse kidney was labeled with Cy3 with the indicated amounts of starting material. RNA was not PEG fractionated. Labeled RNA was combined with a Cy5 reference set and hybridized to the microarray. TIFF images of sections of the arrays are shown, with the positions of three abundant microRNAs in this tissue source. Arrays were scanned at identical PMT voltages. (b) Plots of raw Cy3/Cy5 log-ratios of three microRNAs, with calculated linear regression lines shown (excluding $50 \mu \mathrm{g}$ values, which were saturating). (c) Expanded sections of TIFF images from Figure 1, with the position of mismatched control probes for miR-124a. Individual channels and a composite image are shown. Mismatched miR124a sequences are shown, with mutations in bold. Exact inverse complement sequences were spotted on the array.

the array (Fig. 2a). Abundant microRNAs in this RNA source can be detected in as little as $50 \mathrm{ng}$ total RNA. Plotting un-normalized Cy3/Cy5 ratios against RNA quantity for three microRNAs (Fig. 2b) revealed a linear relationship in the range from $50 \mathrm{ng}$ to $5 \mu \mathrm{g}$. It should be emphasized that for less abundant microRNAs $50 \mathrm{ng}$ does not give adequate signal over background. Using a cutoff value of 1.5-fold over background for raw $\mathrm{Cy} 3$ values, $50 \mathrm{ng}$ total kidney RNA yields detectable signal for $10.5 \%$ of the microRNA probes on the array, whereas $500 \mathrm{ng}$ and $5 \mu \mathrm{g}$ total RNA yields signal for $51 \%$ and $72 \%$ of probes, respectively. Increasing the amount of RNA to $50 \mu \mathrm{g}$ results in partial saturation of signal, likely resulting from competition during the labeling reaction. Removal of large-molecular-weight RNAs by polyethylene glycol (PEG) precipitation extends the linear range to $50 \mu \mathrm{g}$. All clustered data in this report were obtained using $25 \mu \mathrm{g}$ PEG-treated RNA to maximize signal intensity.

Mismatch controls incorporated into the array were used to confirm the specificity of hybridization (Fig. 2c). We cannot exclude the possibility, however, that microRNAs with very similar sequences yield some cross-hybridization. We feel that this does not impair interpretation of the array data. Because microRNAs recognize target mRNAs by hybridization, similar microRNAs will be likely to target overlapping sets of mRNAs. Minimal crosshybridization in our arrays would therefore not affect interpretation of biological pathways regulated by microRNA gene families. Furthermore, the hybridization stringency can be customized for specific melting temperatures of microRNA sequences to optimize specificity. The simple and sensitive array method we describe here facilitates such studies.

Several normalization methods have been developed for microarray data (see ref. 16 for a review). Global median centering multiplies each $\mathrm{Cy} 3 / \mathrm{Cy} 5$ ratio by a constant such that the median value is zero (for log-transformed ratios). This type of normalization method corrects for RNA quantity and quality and the efficiency of individual labeling reactions. It does not perform well, however, when there are large differences in RNA concentrations between samples. More sophisticated methods have been developed, such as Lowess normalization, which takes into account pin-specific biases during the spotting process. We have tested arrays in which all probes are spotted by all pins and have not seen significant pin differences (Pearson correlation $>0.95$ from pin to pin). Another normalization method is based on 'housekeeping' genes that are relatively invariant ${ }^{17}$. The lack of such information about microRNAs precluded such a supervised approach. We therefore used global median centering for all data analysis (see Supplementary Fig. 2 online).

Replicate hybridizations for each RNA source described here showed an average Pearson correlation of 0.915 , with thymus showing the weakest correlation, 0.75 . This reproducibility is more than adequate for the analysis we have performed to date, as individual tissues and cell lines have microRNA expression correlations ranging from 0.0 to 0.7 . Typical correlations among different print batches were in the range of $0.70-0.85$. To maximize data quality, array data from different print batches were not combined in any individual cluster analysis.

\section{Expression analysis of embryonic and adult mouse}

Tissue-specific expression patterns for a subset of known microRNAs have been reported on the basis of on cloning frequencies and northern blotting. To more thoroughly examine expression patterns, we profiled RNA from seven mouse tissues, four different embryonic stages, embryonic stem (ES) cells and embryoid bodies (Fig. 3). Embryoid bodies are ES cells that are cultured in suspension in the absence of leukocyte inhibitory factor (LIF). Such growth conditions promote differentiation into clusters of cells containing all three primordial germ layers, which loosely approximates early development ${ }^{18}$.

Several interesting points are readily apparent in the expression map. The adult tissues separate on the dendrogram from the embryos and from the ES cells and embryoid bodies. The data are complicated by the fact that whole embryos contain mixed cell types, and even tissues are a mixture of several cell lineages. Nevertheless, a large group of microRNAs are highly expressed in diverse adult tissues but are not detectably expressed in embryo. This includes the mir-29 and mir-30 families and the mir-23-mir24-mir-27 polycistronic cluster. The let-7 family, shown in the expanded cluster, is induced at embryonic day (E) 17 and increases in adult tissues. C. elegans let-7 is a well-characterized developmental regulator. The expression pattern in mice suggests this family has a developmental role in this organism as well.

Although the adult tissues cluster together, restricted microRNA expression is apparent for many of them. Clusters of genes that are abundantly expressed in liver, heart, ES cells, brain and thymus are highlighted. Many tissue-specific genes are also expressed in 
Table 1 | Comparison of expression data with published data

\begin{tabular}{|c|c|c|c|}
\hline $\begin{array}{l}\text { Predominant tissue } \\
\text { (microarray) }\end{array}$ & $\begin{array}{c}\text { MicroRNA } \\
\text { gene name }\end{array}$ & Northern data & Cloning data \\
\hline \multirow[t]{2}{*}{ Liver } & $122 a$ & Liver $^{12}$ & Liver \\
\hline & 140 & NA & NA \\
\hline \multirow[t]{7}{*}{ ES cell } & 214 & NA & NA \\
\hline & 134 & NA & NA \\
\hline & 92 & ES cell ${ }^{23}$ & HeLa cell \\
\hline & 25 & NA & HeLa cell \\
\hline & 182 & NA & NA \\
\hline & 204 & NA & NA \\
\hline & 132 & Brain $^{12}$ & NA \\
\hline \multirow[t]{4}{*}{ Thymus } & 210 & NA & NA \\
\hline & $181 a$ & Thymus $^{8}$ & Several \\
\hline & $181 b$ & Thymus $^{8}$ & Several \\
\hline & 212 & NA & NA \\
\hline \multirow[t]{4}{*}{ Heart } & 206 & Heart, skel ${ }^{12}$ & NA \\
\hline & $133 a$ & Heart, skel ${ }^{12}$ & Heart \\
\hline & 1 & Heart, skel ${ }^{12}$ & Heart \\
\hline & 144 & Spleen ${ }^{12}$ & Heart \\
\hline \multirow[t]{15}{*}{ Brain } & 222 & NA & NA \\
\hline & 218 & NA & NA \\
\hline & $125 a$ & Brain $^{12}$ & Kid, skel, brain \\
\hline & $125 b$ & Brain $^{12}$ & Several \\
\hline & $34 a$ & NA & NA \\
\hline & $128 \mathrm{a}$ & Brain $^{12}$ & Brain \\
\hline & 136 & NA & NA \\
\hline & 127 & Brain, spleen ${ }^{12}$ & NA \\
\hline & 138 & NA & NA \\
\hline & 9 & Brain $^{12}$ & Brain \\
\hline & $9 *$ & Brain $^{12}$ & Brain \\
\hline & 219 & NA & NA \\
\hline & $124 a$ & Brain $^{12}$ & Brain \\
\hline & 154 & NA & NA \\
\hline & 149 & NA & NA \\
\hline
\end{tabular}

MicroRNAs that are restricted to one primary tissue, as indicated by microarray data, are listed in the first column. Predominant tissue expression, as indicated by northern blot analysis, is listed in column 2 (based on published work where several tissues were surveyed ${ }^{8,12,23}$ ). Predominant tissues from which microRNAs were cloned are listed in column 3. Only cases where more than three total microRNA clones were reported are listed ${ }^{24}$. NA, not available; skel, skeletal muscle; kid, kidney.

microRNA genes ${ }^{19}$. Rather, our data present an overview of the developmental time course of the microRNA gene family.

To validate our microRNA microarrays, we compared expression profiles to published northern blot and cloning frequency data for microRNAs that are expressed predominantly in one tissue (Table 1). Published data were included in our analysis only if they compared specific microRNA expression levels across several distinct tissues. In general our microarray data correlate very well with published data. The primary difference is the high sensitivity of our method, which allows profiling of weakly expressed genes. In such cases, cloning frequencies are very low, which prevents a good statistical analysis of expression patterns. In the case of northern analysis, the expression levels of many microRNAs are at the detection limit, making quantification unreliable.

\section{DISCUSSION}

We describe here a microarray method for the analysis of microRNA expression levels. We have performed a basic analysis of
124 microRNA genes in different mouse tissues and embryo stages. As previously reported using semiquantitative approaches, microRNAs show tissue-restricted and developmentally restricted expression patterns. We are currently pursuing a more defined developmental and spatial analysis in mouse brain using microdissected samples. In addition, a large number of microRNA genes have been identified since we initially designed our array. We are presently developing a new microarray format that includes 169 human, 184 mouse and 169 rat genes. This includes many brainand ES cell-specific genes that were not included in the array described here.

A microarray method for microRNA profiling has recently been reported ${ }^{20}$. This method differs from the one described here in that it labels and measures quantities of the microRNA primary transcript. Although primary transcript profiling offer some benefits, such as the ability to study the regulation of microRNA transcript processing across many genes at once, it might not provide an exact representation of the expression profile of active mature microRNA species. Because the microRNA primary transcript undergoes several processing and RISC assembly steps before it is in a biologically active form, equating levels of the primary transcript with the mature, RISC-loaded microRNA could be misleading. In addition, labeling the highly structured primary transcript using random primers and Superscript reverse transcriptase may be susceptible to strong biases in efficiency. These biases are likely generated when the reverse transcriptase needs to traverse the highly structured stem-loop to reach the region the probe is directed against. According to a comprehensive study, using gene-specific primers that anneal within the stem-loop and a thermostable reverse transcriptase at high temperature facilitate the labeling of microRNA precursors ${ }^{21}$. In light of these points, and our incomplete understanding of microRNA biogenesis, we feel that direct measurement of mature microRNA species, which are the active form and thus the most relevant indicator of biologic function, is more reliable.

We have performed over 500 arrays spanning six print runs. The ease of use is comparable to that of typical mRNA microarrays. Total unfractionated RNA is a suitable substrate, although using PEG-fractionated RNA allows for a stronger signal. The labeling reaction and cleanup take $2 \mathrm{~h}$. For samples of $5 \mu \mathrm{g}$ or more, a 4-h hybridization is sufficient. It is reasonable to perform 20 microarrays in one day. Because the arrays are produced in-house, costs are kept low.

RNA ligase has a poor reputation for reliability; however, we have had very few failures. The reaction conditions, and the large molar excess of the donor dinucleotide, combine to make the reaction efficient and reliable. The primary source of bias in the labeling reaction is the differential ligation efficiency toward the acceptor nucleotide on the microRNA. An examination of average signal intensities for microRNA probes, grouped by 3 '-terminal nucleotide, showed less than a twofold range.

The sensitivity is much higher than that of current northern blot approaches. For example, we find miR-10b weakly expressed in kidney; however, we can detect this microRNA at 2.7-fold over background using $5 \mu \mathrm{g}$ total RNA. In a published analysis of microRNA expression using a highly sensitive northern blot method (StarFire labeling, Integrated DNA Technologies), no miR-10b expression was detected in any tissue using 1.1-fold over background as a cutoff value ${ }^{12}$. Of course, northern blotting, or the 
recently reported quantitative PCR method, is still essential for verification of microarray data ${ }^{22}$.

The methods we have developed should also be applicable to other non-polyadenylated RNAs, provided a $3^{\prime} \mathrm{OH}$ group is available to ligate the fluorophore. In addition, this method may have utility for direct labeling of mRNAs for standard microarray processes. Direct labeling has the advantage of reduced bias. In fact, because all mRNAs terminate in polyadenosine, ligation biases should be completely eliminated. This fact, coupled with the sensitivity we observed, makes this a promising general approach.

\section{METHODS}

RNA sources. Total RNA from adult mouse tissues was obtained from Ambion. Total RNA from staged mouse embryos was obtained from Clontech. The feederless ES cell line E14Tg2A.4 was grown on gelatin-coated plates in Glasgow MEM (Sigma) supplemented with $2 \mathrm{mM}$ glutamine, $1 \mathrm{mM}$ pyruvate, $1 \times$ nonessential amino acids, $15 \%$ fetal bovine serum (HyClone), $0.1 \mathrm{mM}$ $\beta$-mercaptoethanol and $10^{3}$ units $/ \mathrm{ml}$ leukocyte inhibitory factor (LIF, Chemicon). Embryoid bodies were generated by growth in ES medium lacking LIF and $\beta$-mercaptoethanol on nonadherence Petri dishes. Embryoid bodies were grown in suspension for 3 or $28 \mathrm{~d}$, as indicated in the figure. Total RNA was isolated with Trizol (Invitrogen). High-molecular-weight RNAs were removed by precipitation with $12.5 \%$ PEG-8000, $1.25 \mathrm{M} \mathrm{NaCl}$. For the experiments shown in Figure 1, RNA was fractionated on a $15 \%$ acrylamide, $8 \mathrm{M}$ urea TAE gel and extracted in water. For experiments shown in the remaining figures, RNA was only fractionated with PEG.

Nucleic acid labeling. RNA labeling was performed using RNA ligase as described ${ }^{14}$. The labeling reaction contained RNA (typically $25 \mu \mathrm{g}$ before PEG precipitation), $0.1 \mathrm{mM}$ ATP, $50 \mathrm{mM}$ HEPES, pH 7.8, $3.5 \mathrm{mM}$ DTT, $20 \mathrm{mM} \mathrm{MgCl}_{2}, 10 \mathrm{mg} / \mathrm{ml} \mathrm{BSA}$, $10 \%$ DMSO, and 500 ng 5'-phosphate-cytidyl-uridyl-Cy3-3' (Dharmacon) with 20 units T4 RNA ligase (NEB). The labeling reaction was allowed to proceed at $0{ }^{\circ} \mathrm{C}$ for $2 \mathrm{~h}$. Labeled RNA was precipitated with $0.3 \mathrm{M}$ sodium acetate, 2 volumes ethanol, and resuspended in water containing the labeled reference set. Reference-set oligodeoxynucleotides complementary to each probe were synthesized (Sigma-Genosys). A $0.5 \mathrm{mM}$ mixture of all reference oligonucleotides was labeled using the Alexa 647 (Cy5) Ulysis kit from Molecular Probes, and excess labeling reagent was removed by Sephadex G-25 spin column (Amersham). This reference was used at a 1,000-fold dilution in each hybridization mixture.

Microarray methods. Oligonucleotide probes for 124 microRNAs (see Supplementary Fig. 1 online for sequences) were synthesized (Sigma-Genosys) in duplicate and duplicate spotted on Corning GAPS-2 slides in $3 \times$ SSC using a Genomic Solutions OmniGrid 100 arrayer. Slides were crosslinked with UV at $600 \mathrm{~mJ}$, prehybridized in $3 \times$ SSC, $0.1 \%$ SDS, $0.2 \%$ BSA for $45 \mathrm{~min}$, and hybridized for $2 \mathrm{~h}$ at $37{ }^{\circ} \mathrm{C}$ in $400 \mathrm{mM} \mathrm{Na} 2 \mathrm{HPO}_{4} \mathrm{pH} 7.0$, $0.8 \%$ BSA, $5 \%$ SDS, $12 \%$ formamide. Hybridization was performed in disposable chambers from MJ Research (part number SLF-0601). These consist of square frames of interior dimension $15 \mathrm{~mm} \times 15 \mathrm{~mm}$ that are made of an adhesive polymer. They are attached to the slide with the array inside the frame. A plastic coverslip is placed on the upper side, creating a chamber of $65 \mu \mathrm{l}$ volume. The hybridization mixture, total volume $61 \mu \mathrm{l}$, is injected into the chamber. Slides were washed once in $2 \times$ SSC, $0.025 \%$ SDS, three times in $0.8 \times$ SSC, and three times in $0.4 \times \mathrm{SSC}$, at $25{ }^{\circ} \mathrm{C}$. Slides were scanned with a Genepix 4000B Scanner (Axon) and raw pixel intensities extracted with Axon software.

Computational methods. $\mathrm{Cy} 3$ and $\mathrm{Cy} 5$ median pixel intensity values were background subtracted, and $\mathrm{Cy} 3 / \mathrm{Cy} 5$ ratios were obtained. Data points were removed when Cy5 values did not exceed 200\% background. Database calculations were performed with Winstat for Excel. Cy3/Cy5 ratios were log-transformed (base 2 ), median centered by arrays and genes, and hierarchically clustered (average linkage correlation metric) using the Cluster program from Stanford University. Dendrograms and expression maps were generated by Treeview from Stanford.

Accession numbers. All microarray data were deposited at GEONCBI with the following accession numbers: GSE1635 and GSM28499-GSM28533.

Note: Supplementary information is available on the Nature Methods website.

\section{ACKNOWLEDGMENTS}

Cluster and Treeview software were developed by M. Eisen and generously distributed by Stanford University. The authors acknowledge the UNC Genomics and Microarray Core Facility for assisting in microarray production. The authors thank G. Hannon, Y. Xiong, M. Carmell, and members of the Hammond laboratory for critical reading of the manuscript and discussion of the project. S.M.H. is a General Motors Cancer Research Foundation Scholar.

\section{COMPETING INTERESTS STATEMENT}

The authors declare that they have no competing financial interests.

\section{Received 6 June; accepted 10 August 2004}

Published online at http://www.nature.com/naturemethods/

1. Bartel, D.P. MicroRNAs: genomics, biogenesis, mechanism, and function. Cell 116, 281-297 (2004).

2. Yekta, S., Shih, I.H. \& Bartel, D.P. MicroRNA-directed cleavage of HOXB8 mRNA. Science 304, 594-596 (2004).

3. Doench, J.G., Petersen, C.P. \& Sharp, P.A. siRNAs can function as miRNAs. Genes Dev. 17, 438-442 (2003).

4. Zeng, Y., Yi, R. \& Cullen, B.R. MicroRNAs and small interfering RNAs can inhibit mRNA expression by similar mechanisms. Proc. Natl. Acad. Sci. USA 100, 97799784 (2003).

5. Arasu, P., Wightman, B. \& Ruvkun, G. Temporal regulation of lin-14 by the antagonistic action of two other heterochronic genes, lin-4 and lin-28. Genes Dev. 5, 1825-1833 (1991).

6. Reinhart, B.J. et al. The 21-nucleotide let-7 RNA regulates developmental timing in Caenorhabditis elegans. Nature 403, 901-906 (2000).

7. Vella, M.C., Choi, E.Y., Lin, S.Y., Reinert, K. \& Slack, F.J. The C. elegans microRNA let-7 binds to imperfect let-7 complementary sites from the lin-41 $3^{\prime}$ UTR. Genes Dev. 18, 132-137 (2004).

8. Chen, C.Z., Li, L., Lodish, H.F. \& Bartel, D.P. MicroRNAs modulate hematopoietic lineage differentiation. Science 303, 83-86 (2004).

9. Aravin, A.A. et al. The small RNA profile during Drosophila melanogaster development. Dev. Cell 5, 337-350 (2003).

10. Sempere, L.F., Sokol, N.S., Dubrovsky, E.B., Berger, E.M. \& Ambros, V. Temporal regulation of microRNA expression in Drosophila melanogaster mediated by hormonal signals and broad-complex gene activity. Dev. Biol. 259, 9-18 (2003).

11. Lim, L.P. et al. The microRNAs of Caenorhabditis elegans. Genes Dev. 17, 991-1008 (2003).

12. Sempere, L.F. et al. Expression profiling of mammalian microRNAs uncovers a subset of brain-expressed microRNAs with possible roles in murine and human neuronal differentiation. Genome Biol. 5, R13 (2004). 
13. Krichevsky, A.M., King, K.S., Donahue, C.P., Khrapko, K. \& Kosik, K.S. A microRNA array reveals extensive regulation of microRNAs during brain development. RNA $\mathbf{9}$, 1274-1281 (2003).

14. Igloi, G.L. Nonradioactive labeling of RNA. Anal. Biochem. 233, 124-129 (1996).

15. Griffiths-Jones, S. The microRNA Registry. Nucleic Acids Res. 32, D109-D111 (2004).

16. Quackenbush, J. Microarray data normalization and transformation. Nat. Genet. 32 (Suppl.), 496-501 (2002).

17. Wang, Y., Lu, J., Lee, R., Gu, Z. \& Clarke, R. Iterative normalization of cDNA microarray data. IEEE Trans. Inf. Technol. Biomed. 6, 29-37 (2002).

18. Eistetter, H.R. A mouse pluripotent embryonal stem cell line stage-specifically regulates expression of homeo-box containing DNA sequences during differentiation in vitro. Eur. J. Cell Biol. 45, 315-321 (1988).
19. Leahy, A., Xiong, J.W., Kuhnert, F. \& Stuhlmann, H. Use of developmental marker genes to define temporal and spatial patterns of differentiation during embryoid body formation. J. Exp. Zool. 284, 67-81 (1999).

20. Liu, C.G. et al. An oligonucleotide microchip for genome-wide microRNA profiling in human and mouse tissues. Proc. Natl. Acad. Sci. USA 101, 9740-9744 (2004).

21. Schmittgen, T.D., Jiang, J., Liu, Q. \& Yang, L. A high-throughput method to monitor the expression of microRNA precursors. Nucleic Acids Res. 32, e43 (2004).

22. Meister, G. et al. Human argonaute2 mediates RNA cleavage targeted by miRNAs and siRNAs. Mol. Cell 15, 185-197 (2004).

23. Houbaviy, H.B., Murray, M.F. \& Sharp, P.A. Embryonic stem cell-specific microRNAs. Dev. Cell 5, 351-358 (2003).

24. Lagos-Quintana, M., Rauhut, R., Lendeckel, W. \& Tuschl, T. Identification of novel genes coding for small expressed RNAs. Science 294, 853-858 (2001). 